\title{
MATERIALS INSTABILITIES AND IMAGE SEGMENTATION: NEW TRENDS IN PDES AND IN THE CALCULUS OF VARIATIONS
}

\author{
Irene Fonseca ${ }^{1,2}$ \\ 'Department of Mathematical Sciences, Carnegie Mellon University, Pittsburgh, PA 15213, \\ ${ }^{2}$ Max-Planck Institut für Mathematik in den Naturwissenschaften, Leipzig, Germany
}

\begin{abstract}
In recent years remarkable advances in technology have lead to the mathematical study of materials instabilities such as phase transitions and twinning, the onset of microstructure, the formation of grain boundaries, nucleation of phases, defectiveness in certain crystalline "smart" materials. The need to understand and predict macroscopic behavior from microscopic to mesoscopic data, and questions related to optimal design and homogenization of composite materials and very thin films have opened new areas of mathematics still almost unexplored. As it turns out, mathematical models for equilibria and dynamical evolution of phase boundaries for these materials fall outside the scope of classical theories, mostly due to the fact that the underlying energies are nonconvex. Although different in motivation, the mathematical difficulties encountered in image segmentation in computer vision are essentially the same as in the study of fracture and damage for these materials. Any progress in the understanding of such nonlinear phenomena will entail the introduction and development of new mathematical tools.
\end{abstract}

\section{Background}

The study of materials instabilities such as phase transitions, nucleation and growth, defectiveness, hysteresis, and the onset of microstructure incertain (shape memory) alloys, the need to understand properties of very thin ferromagnetic films and other interesting nonlinear material phenomena lead us to new areas of mathematics in Partial Differential Equations and in the Calculus of Variations. Quasistatic and evolutionary or dynamical mathematical analysis of these problems fall outside the scope of classical theories, and so innovative, more powerful methods must be introduced.

Generally, some technologically important properties of smart materials are related to the multiple-well structure of the energy densities, and in simplified models equilibria may be found by searching minima for a total energy

$$
\mathfrak{J}: u \mapsto \int_{\Omega} f(x, u, D u) d x+\ldots
$$

where $\Omega \subset \mathrm{R}^{N}$ is a bounded, open domain (usually $N=3$ and $\Omega$ represents the reference 
configuration), $u: \Omega \rightarrow \mathrm{R}^{d}$ is in the class of admissible fields (e.g. $d=3$ and $u$ stands for the deformation of the body in nonlinear elasticity, while $d=2$ in the study of thin films), and $f: \Omega \times \mathbf{R}^{d} \times \mathbb{M} \mid d \times N \rightarrow \mathbf{R}$ is the bulk energy density, $\mathbb{M}^{d \times N}$ denotes the space of $d \times N$ matrices. The remaining terms in the total energy $\mathfrak{I}$ may account for loads, body forces, surface or edge energies, etc. (see [26], [27]).

Following Gibbs' criterion for equilibria, it is well known that (see [7], [30]) existence of minimizers for $\mathfrak{I}$ is related to "convexity" properties of $\xi \rightarrow f(x, u, \xi)$, for each fixed $(x, u) \in \Omega \times \mathbf{R}^{d}$. In turn, development of microstructure is tied up with the oscillatory behavior of the gradients of minimizing sequences and with the multiple well structure of $\xi \rightarrow f(x, u, \xi)$, so all the classical techniques of Convex Analysis and of the theories of partial differential equations or systems that do not change type cannot be applied to this setting. In addition, we may be required to consider constrained problems (e.g. in the study of magnetic materials or liquid crystals $|u|$ must be constant, see [6], [14], [18], [24], [29]), and macroscopic cracking, damage and fracture may impose discontinuities on the fields $u$ (see [19]). The latter is also the case for image segmentation analysis in computer vision, where some of the models adopted (e.g. Mumford and Shah [11], [16], [31]) are very similar to Griffith's models for fracture of elastic materials. Dealing with nonconvex energies and with discontinuous fields brings in major mathematical challenges, and although new tools have been introduced to tackle some of these problems, general knowledge in these areas is still quite incipient.

\section{Current Work}

Here we focus on the three questions below and we outline some of the results already obtained.

Question 1 : Can we find necessary and sufficient conditions on the energy densities guaranteeing lower semicontinuity of the energy $\mathfrak{I}$, i.e.

$$
u_{n} \rightarrow u \Rightarrow \mathfrak{I}(u) \leq \liminf _{n \rightarrow \infty} \mathfrak{I}\left(u_{n}\right) ?
$$

Here the convergence $u_{i r} \rightarrow u$ is taken in the "weak" sense, i.e. we allow oscillatory behavior of the sequence $\left\{\nabla u_{n}\right\}$ which converges to $\nabla u$ in average over subsets of $\Omega$. Depending on the growth conditions of $f$ and on the general framework of the problem, the mathematical space where this convergence takes place may vary. As an example, in nonlinear elasticity generally we consider Sobolev spaces

$$
W^{1, p}(\Omega):=\left\{u: \Omega \rightarrow \mathbf{R}^{d}: u \in L^{p}\left(\Omega ; \mathbf{R}^{d}\right), D u \in L^{p}\left(\Omega ;\left.\mathbb{M}\right|^{d \times N}\right)\right\},
$$

where $L^{p}\left(\Omega ; \mathbf{R}^{k}\right):=\left\{v: \Omega \rightarrow \mathbf{R}^{k}: \int_{\Omega}|v|^{p} d x<+\infty\right\}, 1 \leq p<+\infty$, and where the generalized notion of (distributional) derivative $D u$ is to be understood in the sense of integration by parts, i.e. $D u$ is that matrix-valued Radon measure $\mu$ such that 


$$
\int_{\Omega} u_{i} \frac{\partial \varphi}{\partial x_{j}} d x=-\int_{\Omega} \varphi d \mu_{i j}, j=1, \ldots, N, i=1, \ldots, d,
$$

for all smooth function with compact support in $\Omega, \varphi \in C_{s}^{\infty}(\Omega)$. On the other hand, in the study of fracture or in the analysis of image segmentation problems the function $u$ may have jumps, and a function space commonly used has been proposed by Ambrosio and De Giorgi [4], the space $S B V$ of functions of special bounded variation. Here the distributional derivative $D u$ is a Radon measure which can be decomposed as

$$
D u=\nabla u,{ }^{N}+\left(u^{+}-u^{-}\right) \otimes \mathscr{H}^{N-1}\lfloor S(u)
$$

where,$^{N}$ stands for the Lebesgue measure in $\mathrm{R}^{N}, \nabla u$ is the density of the measure $D u$ with respect to the Lebesgue measure, $S(u)$ is the (N-1 dimensional) jump set of $u, u^{+}$and $u^{-}$are the traces of $u$ on each side of $S(u), v$ is a unit normal to $S(u)$, and $H^{N-1}\lfloor S(u)$ is the (surface) $N-1$ Hausdorff measure restricted to the jump set. The energy $\mathfrak{I}$ may take the form

$$
\mathfrak{I}(u)=\int_{j \Omega} f(x, u, \nabla u) d x+\int_{S(u)} \psi\left(x, u^{+}, u^{-}, v\right) d H^{N-1}(x)+\ldots
$$

in order to account for interfacial energy contributions.

The answer to Question 1 is relevant to the study of existence of minima as, due to Direct Method of the Calculus of Variations, existence of minima for $\mathfrak{I}$ is guaranteed provided the class of energetically bounded admissible fields $u$ is weakly compact and that lower semicontinuity holds. It is known (see [1]) that in the case of Sobolev spaces lower semicontinuity is satisfied if $0 \leq f(x, u, \xi) \leq a(x, u)\left(1+|\xi|^{p}\right)$ for some locally bounded function $a$, and if $f$ is quasiconvex, i.e.

$$
f\left(x_{0}, u_{0}, \xi\right) \leq \int_{Q} f\left(x_{0}, u_{0}, \xi+D \varphi(x)\right) d x
$$

for all $\varphi \in C_{\jmath}^{\infty}\left(Q ; \mathbf{R}^{d}\right), Q:=(0,1)^{N}$ (similar results have been obtained in $S B V$ and in more general settings, see [3])) . This latter property means, essentially, that affine mappings are energetically preferred amongst all that deform the unit cube keeping the affine Dirichlet boundary conditions $x \in \partial Q \mapsto \xi x$.

Question 2 : Suppose that we know how to fully characterized lower semicontinuous functional, that the functional under consideration fails to meet the criteria, and that given a macroscopic state $u$, we search for the its effective or relaxed energy, i.e.

$$
\boldsymbol{\zeta}(u):=\inf _{\left\{u_{n}\right\}}\left\{\liminf _{n \rightarrow \infty} \mathfrak{I}\left(u_{n}\right): u_{n} \#\right\} .
$$


Clearly, due to lack of lower semicontinuity, it may happen that $\mathfrak{I}(u)>\boldsymbol{\delta}(u)$, however it may be shown (see [17]) that the initial problem and the relaxed one admit the same minimizing sequences,

$$
\inf _{u} \mathfrak{I}(u)=\min _{u} \delta(u)
$$

Question 2 concerns the finding of an integral representation for $\boldsymbol{\delta}$, i.e. the search for an effective bulf energy $F$ and a full characterization of the remaining terms of

$$
\boldsymbol{\zeta}(u)=\int_{\Omega} F(x, u, \nabla u) d x+\mu_{s}
$$

where $\mu_{s}$ may be a singular measure which is produced via the relaxation procedure and it accounts for defectiveness, the onset of cracks, etc.

In the case where

$$
\mathfrak{I}(u)=\int_{\Omega} f(x, u, \nabla u) d x
$$

and $f$ is a Caratheodory function, $0 \leq f(x, u, \xi) \leq a(x, u)\left(1+|\xi|^{p}\right)$, where $a$ is a locally bounded function, it can be shown that (see $[\mathrm{AF}],[\mathrm{D}])$

$$
\zeta(u)=\int_{\Omega} Q f(x, u, D u) d x
$$

where the quasiconvexification $Q f$ is defined by

$$
Q f\left(x_{0}, u_{0}, \xi\right):=\inf \left\{\int_{Q} f\left(x_{0}, u_{0}, \xi+D \varphi(x)\right) d x: \varphi \in C_{0}^{\infty}\left(\Omega ; \mathbf{R}^{d}\right)\right\} .
$$

Ouestion 3 : As mentioned previously, relaxation methods are particularly useful when dealing with minimizers or with minimizing sequences, since these are the same for the initial or the relaxed energies. Suppose, however, that $\left\{u_{n}\right\}$ is not a minimizing sequence (e.g. its macroscopic limit could be simply metastable), and that we want to identify the limiting nonlinear energy

$$
\mathfrak{I}\left(u_{n}\right) \rightarrow ?
$$

A mathematical tool successfully used to answer this question is the notion of generalized solutions, such as Young measures and H-measures (see [8], [32], [33]). Although Young measures habe been used for quite some time in Control Theory and Optimization, they were first introduced in Partial Differential Equations by Tartar [32] in order to relate the information obtained from linear balance equations with the information resulting from pointwise nonlinear constitutive relations. Here, associated to an oscillating se-quence of 
gradients $\left\{D u_{n}\right\}$ there is a parametrized family of probability measures $\left\{v_{r}\right\}_{r c o}$ (Young measure) which gives local statistics of the sequence and, in particular, it may describe nonlinear limits

$$
\left.\lim _{n \rightarrow \infty} \int_{\Omega} f\left(x, u_{n}, D u_{n}\right) d x=\right\rfloor_{\Omega} \int_{M^{\alpha \times N}} f(x, u(x), \xi) d v_{\times}(\xi) d x .
$$

A considerable research effort has been dedicated during the last few years to obtaining the characterization of such probability measures, so that, for example, given a priori certain statistics (such as twinning pattern) we may be able to decide beforehand whether or not they can be physically realized by a certain material with a known microscopic or mesoscopic distribution. This question has been fully answered in the context of nonlinear elasticity (see [9], [28]), and also in the case where they may be some defectiveness or concentrations of the material (see [21]).

\section{Conclusions and Future Directions}

Modern technology and the development of new materials present non trivial challenges to the mathematical understanding of the nonlinear phenomena involved. New theories must be introduced, opening entire novel areas of research in mathematical sciences. Although some insight has been obtained during the last few years, there is still an enormous task ahead in order to fully master the study of materials instabilities.

A strong research investment is being placed nowadays on the study of phase transitions (see [26], [34], [35]), ferromagnetic (active) thin films (see [7], [25]), fracture and damage (see $[19,20]$ ), the passage from microscopic to macroscopic levels, the propagation and evolution of microstructure (see [13], [23]), the dynamical evolution of phase boundaries (see [15]), relaxation theories (see [12]), of the regularity and evolution of the crack in a brittle continuous medium and related questions in image segmentation in computer vision (see [2], [5]), constrained variational problems (liquid crystals and magnetic materials), crystals with defects (dislocations) (see [22]), liquid crystals with defects (disclinations), optimal design and homogenization (see [20]).

Acknowedgments: This work was partially supported by the National Science Foundation through the Center for Nonlinear Analysis, by the National Science Foundation under grant No. DMS--9500531, and by the MaxPlanck Institute for Mathematics in the Sciences in Leipzig, Germany.

\section{References}

[1] Acerbi, E. and N. Fusco, "Semicontinuity problems in the calculus of variations", Arch. Rat. Mech. Anal. 86 (1984), 125 - 145.

[2] Acerbi, E, I. Fonseca and N. Fusco, "Regularity results for equilibria in a variational model for fracture". To appear in Proc. R. Soc. Edin.

[3] Ambrosio L., "On the lower semicontinuity of quasi-convex integrals in $S B V\left(\Omega ; \mathrm{R}^{k}\right)$ ", 
Nonlinear Anal. 23 (1994), 405-425.

[4] Ambrosio, L. and E. De Giorgi, "Un nuovo tipo di funzionale del calcolo delle variazioni", Atti. Accad. Naz. Lincei Rend. Cl. Sci. Fis. Mat. Natur. 82 (1988), 199210.

[5] Ambrosio, L., N. Fusco and D. Pallara, "Partial regularity of free discontinuity sets II", Preprint Dip. Mat. e Appl. Napoli, (1995).

[6] Ambrosio, L. and E. Virga, "A boundary-value problem for nematic liquid crystals with variable degree of orientation", Arch. Rat. Mech. Anal. 114 (1991), 335-347.

[7] Ball, J. M., "Convexity conditions and existence theorems in nonlinear elasticity", Arch. Rat. Mech. Anal. 63 (1977), 337-403.

[8] Ball, J. M., "A version of the fundamental theorem for Young measures", in $P D E$ 's and. Continuum Models of Phase Transitions, Lecture Notes in Physics 344 (Rascle, M., D. Serre, M. Slemrod, eds.), Springer, 207-215.

[9] Ball, J. M. and James, R. D. "Fine phase mixtures as minimizers of energy", Arch. Rat. Mech. Anal., 100 (1987), 13-52.

[10] Bhattacharya, K. and R. James, "Thin films of martensitic materials with applications to microactuators", submitted to J. Mech. Phys. Solids.

[11] Blake, A. and A. Zisserman, Visual Reconstruction, MIT Press, Boston, 1987.

[12] Bouchitte, G., I. Fonseca and L. Mascarenhas, "A global method for relaxation". Submitted to Arch. Rat. Mech. Anal.

[13] Brandon, D., I. Fonseca, P. J. Swart, "The creation and propagation of oscilla-tory microstructure in a dynamical model of displacive phase transformations", in Progress in partial Differential Equations: the Metz Surveys 3, Pitman Res. Notes Math. Ser., 314, Longman Sci. Tech., Harlow, 1994, 130-144.

[14] Brown, W. F., Micromagnetics, J. Willey \& Sons, 1963.

[15] Cahn, J. W. and J. E. Taylor, "Surface motion by surface diffusion", preprint.

[16] Carriero, M., E. De Giorgi and A. Leacci, "Existence theorem for a minimum problem with free discontinuity set", Arch. Rat, Mech. Anal. 108 (1989), 195-218.

[17] Dacorogna, B., Direct Methods in the Calculus of Variations, Applied Math. Sciences 78, Springer-Verlag, 1989.

[18] Ericksen, J., "Liquid crystals with variable degree of orientation", Arch. Rat. Mech. Anal. 113 (1991), 97-120.

[19] Fonseca, I. and G. Francfort, "Relaxation in $B V$, versus quasiconvexification in $W^{1, p}$; a model for the interaction between fracture and damage", Calc. Var. 3 (1995), 407-446.

[20] Fonseca, I. and G. Francfort, "Optimal design problems for membranes and thin films", in preparation.

[21] Fonseca, I., S. Müller and P. Pedregal, "Analysis of concentration and oscillation effects generated by gradients". To appear in SIAM J. Mathematical Analysis.

[22] Fonseca, I. and G. Parry, "Equilibrium configurations of defective crystals", Arch. Rat. Mech. Anal. 120 (1992), 245-283.

[23] Fonseca, I., J. Schaeffer and M. Shvartsman, "Oscillations in one-dimensional elasticity with surface energy". To appear in Quaterly of Applied Mathematics. 
[24] Gioia, G. and R. D. James, "Micromagnetics of very thin films", submitted to Proc. $R$. Soc. Edin.

[25] Gioia, G. and M. Ortiz, "Delamination of compressed thin films". To appear in $A d v$. Appl. Mech.

[26] Gurtin, M. E., "On a theory of phase transitions with interfacial energy", Arch. Rat.Mech. Anal. 87 (1984), 187-212.

[27] Herring, C., "Some theorems on the free energies of crystal surfaces", Phys. Rev. 82 (1951), 87-93.

[28] Kinderlehrer, D. and P. Pedregal, "Gradient Young measures generated by sequences in Sobolev spaces", J. Geom. Anal., 4, 59-90, 1994.

[29] Lin, Fang-Hua, "Nonlinear theory of defects in nematic liquid crystals; phase transition and flow phenomena", Comm. Pure and Appl. Math. 42 (1989), 789-814.

[30] Morrey C.B., Multiple Integrals in the Calculus of Variations, Springer-Verlag, Berlin, 1966.

[31] Mumford, D., and J. Shah, "Optimal approximations by piecewise smooth functions and associated variational problems", Comm. Pure and Appl. Math 42 (1989), 577-685.

[32] Tartar, L., "Compensated compactness and applications to partial differential equations", R. Knops (ed.), Pitman Res. Notes Math. 39 (1979), 136-212.

[33] Tartar, L., "H-measures, a new approach for studying homogenisation, oscillations and concentration effects in partial differential equations", Proc. Roy. Soc. Edin. 115A (1990), 193-230.

[34] Truskinovsky, L., "Nucleation and growth in elastodynamics", to appear in Dynamics of Crystal Surfaces and Interfaces (P. M. Duxbury, T. Pence eds.), Fundamental Materials Science Series, Plenum, 1997.

[35] Visintin, A., Models of Phase Transitions, Birkhäuser, 1996. 
